\title{
Social-Cultural Factors and Complementary Feeding of Children 6-23 Months Among the Maasai in Narok South Kenya.
}

\author{
Stellah Malaso Koini ${ }^{1}$ (Corresponding author) \\ School of Nursing, Maasai Mara University \\ PO BOX 861-20500, Narok, Kenya.
}

Tel: +254720722948 E-mail: lollymalaso@gmail.com or kstellah@mmarau.ac.ke

Sophie Ochola ${ }^{2}$

School of Applied Science, Kenyatta University

Nairobi, Kenya.

Tel: +254 721449803 E-mail: ocholasa55@gmail.com

\author{
Irene $\mathrm{A} \mathrm{Ogada}^{3}$ \\ Department of Human Nutrition \\ St. Francis Xavier University \\ Tel: +254 723955466 E-mail: tujunz@yahoo.com
}

\begin{abstract}
Background: The Maasai socio-cultural beliefs and practices have been shown to influence their infant feeding hence determines their nutritional status.

Objective: This study aimed to determine the complementary feeding practices among the Maasai community in Narok South sub-County.

Methodology: A cross-sectional analytical study design was adopted. A total sample of 400 mothers and children were selected using simple random sampling. Data was collected using an infant and young child WHO semistructured questionnaire and focus group discussion (FGDs) guides. Anthropometric data was interpreted using Zscores with reference to the World Health Organization (WHO) 2006. ENA for SMART, 2011 software was used to analyze anthropometric data. Quantitative data was analyzed using statistical packages for social sciences (SPSS) version 20. Statistical significance was set at $p$ value less than 0.05 .

Results: Early introduction of complementary feeding was common in the community. Comparison of the prevalence of malnutrition between the boys and girls revealed that stunting and underweight was significantly higher in the girls (stunting, 33.3\%; underweight, 14.1\%) compared to the boys (stunting, 26.8\%; underweight, $11.3 \%)$, On the other hand a larger proportion of the boys had low weight for height $(8.9 \%)$ compared with the girls $(11.0 \%)$. Cultural factors and taboos were seen to have greatly influenced the infant feeding practices.

Conclusion: Socio-cultural factors associated with the complementary feeding have a strong influence on children 6-23 months of age in the Maasai community, in Narok South -Sub County.

Keywords: socio-cultural factors, complementary feeding, Nutrition status, pastoralists.

DOI: $10.7176 / \mathrm{JHMN} / 62-18$

Publication date:May $31^{\text {st }} 2019$

\section{Introduction}

Complementary feeding is greater determinant towards child growth and development, this is sometimes not met due to false beliefs. Proper infant feeding practices which include Breastfeeding is the most effective intervention to save children's lives and could prevent $13 \%$ of all deaths among children less than five years if practiced optimally, while good complementary feeding could prevent another $6 \%$ of deaths. Introduction of safe complementary foods is practiced by $67 \%$ of mothers at 6 months worldwide. (UNICEF/WHO, 2015). There is a need for promotion and encouragement of optimal feeding practices for improving health of children. In fact, beliefs, attitudes, and practices surrounding foods and eating are some of the most important components of cultural identity, therefore, it is not surprising that in multicultural societies, great variability exists in the cultural characteristics of the diet.
\end{abstract}

Nutritionally appropriate and safe complementary foods should be introduced after six months. Breastfeeding should be encouraged for up to two years of age or longer. When implemented in both developing and developed 
countries, these recommendations have been shown to significantly reduce infant morbidity. Malnutrition contributes to more than half of all under-five childhood deaths throughout the developing world; and has been responsible directly or indirectly for about $60 \%$ of the 10.9 million deaths annually of which over two-thirds of these deaths; are often associated with inappropriate feeding practices, occurring during the first year of life. The associated effects of poverty, inadequate household access to food, infectious disease, and inadequate breastfeeding and complementary feeding practices often lead to illness, growth faltering, nutrient deficiencies, delayed development, and death, particularly during the first two years of life are a major obstacle to sustainable socio-economic development and poverty reduction. Any food provided to a newborn before the initiation of mother's breastfeeding is considered to be a pre-lacteal feed. The type of pre-lacteal feeds depends on the culture. It may include ghee (refined butter), herbs, honey, sugar, sugar juice water etc. Introduction of pre-lacteal feeds is a known barrier to continuation of exclusive breastfeeding. Promoting, protecting and supporting optimal nutrition is relevant to life-long health and require not only an understanding of physiological and biochemical facets but an appreciation of the strong social and cultural influences. Overall the study will inform policy makers of appropriate evidence based interventions from county to national level of policy making, ensuring optimal outcomes for children 6-23 months.

\section{Methods}

A cross-sectional analytical study design was adopted, with mixed methods (quantitative and qualitative techniques) for data collection, analysis and presentation. The design was selected because it allows for interrelationship between study variables at one point in time. The study was conducted in Narok South Sub-County children teaching hospital. Four hundred mothers during their admissions or visiting advisory clinic of the hospital were selected to participate in this study. The inclusions criteria were: the respondent has to be the infant' s mother, has an infant not older than 2 years of age at the time of the study and has a willing to participate in the study. Infant and Young Child Feeding, (IYCF) tested questionnaire was used to collect information on feeding attitudes and practices of their infants in relation to their beliefs and a range of socio economic factors. Data were then exported to statistical package for social science (SPSS). Informed consent was taken from participating mothers. The researcher administered and collected data from the respondents. All the 400 mothers were interviewed, the average interview duration per respondent was 20 minutes to a half an hour. The data collected were edited, coded and analyzed for common themes, including multi-variants chi-square and proportions to look at the various relationships that exist among variables of interest.

\section{Results}

A total of 400 participants were included in the study and were residents of Narok South Sub County. Majority of the households in the study sample $(91.2 \%)$ were male headed; female headed household accounted only for $8.8 \%$. There were more female infants (50.5\%), than males (49.5\%). In this study, half of the mothers/caregivers $(53 \%)$ of the responded that complementary foods should be introduced at 6 months while the rest $47 \%$ feel it should be introduced at 4 months. Among the subject in this study however, breastfeeding was rarely practiced an hour after birth but rather delayed by majority $(82.8 \%)$. Only (17.2\%) put their babies on breast within an hour after birth, $(42.5 \%)$ within 24 hours with the rest $(40.3 \%)$ giving the first breast feed more than 24 hours after delivery. By the third day after delivery majority of the women in the study $(93.8 \%)$ reported that they had put their babies on breast while only $(6.3 \%)$ had not. all the responders $(100 \%)$ agreed that indeed breastfeeding was an important practice. However, only about half $(49.1 \%)$ of the caregivers believe that a child can survive on breast milk alone for the first six months. Comparison of the prevalence of malnutrition between the boys and girls revealed that stunting was significantly higher in the girls (stunting, 33.3\%; and boys (stunting, 26.8\%; while underweight, was the opposite in the two sexes $22.9 \%$ ) of the girls were underweight, lower than their male children $35.9 \%$ ). On the other hand, a bigger proportion of the boys had higher weight - for- height $(9.1 \%)$ compared with the girls $(3.0 \%)$. Although mothers showed positive attitude towards timely introduction of infant feeding $\left(\mathrm{M}_{4} \mathrm{FGD}_{3} 2016\right)$ this was not the case as confirmed in the FGDs, the mothers said, "We give cow's milk to our children as we have always done, it's our culture". The cow's milk was diluted using boiled herbs. "We ensure milk is safe by putting herbs in the milk."

Although most of the mothers confirmed that they received different advices from their traditional perception others avoided this practices.

"The cultural practices in the community bind us to feed our children using cow milk and additional herbs which are given to babies to clean their stomach and prevent them from diseases." $\left(\mathrm{M}_{5} \mathrm{FGD}_{3} 2016\right.$.) This was a statement from one of the mothers.

Mothers who practiced exclusive breastfeeding and timely introduction of complementary feeding reported to have received information from healthcare practitioners during their antenatal clinic visits, those who practiced timely 
complementary feeding it was not complicated and they saw its benefits as their children rarely developed stomach infections or diarrhea. Some mothers who did not practiced exclusive breastfeeding and adequate, timely complementary feeding said the child become sick and eventually died from diarrhea.

Another caregiver said she fed the child because the mother had gone away for long and hence the infant was hungry. "I fed the baby with milk the mother was away for long and the child was crying," ( $\mathrm{M}_{1} \mathrm{FGD}_{3} 2016$.)

Most mothers/caregivers agreed that poor complementary was widely practiced by the community because of cultural diversity and lifestyle. "Some mothers go herding cows or sheep therefore it is difficult to exclusively breastfeed, we introduce children to cow's milk and this is our culture." $\left(\mathrm{M}_{2} \mathrm{FGD}_{3} 2016\right.$.)

Another had this to say, "I would like to follow what doctors and nurses tell me but I have other children to feed, I have to go to the market." $\left(\mathrm{M}_{3} \mathrm{FGD}_{3} 2016\right)$. This was confirmed by many mothers from findings of this study. Timely complementary feeding was rarely practiced and was confirmed by the FGDs.

Nutrition education in the hospitals and clinics was evident as another mother in the FGDs confirmed "I was taught how to exclusively breastfeed for six months and start other foods after six months with continued breastfeeding the child will become well and healthy." ( $\left.\mathrm{KII}_{4} 2016.\right)$ "I was told not to give water; porridge or milk and Ifollowed that." ( $\left.\mathrm{M}_{3} \mathrm{FGD}_{4} 2016.\right)$.

Socio-cultural determinants of child feeding practices as described by the respondents included Early marriage, as mentioned by $80.3 \%$ of the respondents. This is followed by child care practices and religious/spiritual practices as mentioned by $50.4 \%$ and $41.5 \%$ of the respondents respectively.

Figure 1. Who makes decision on when to start complementary feeding.

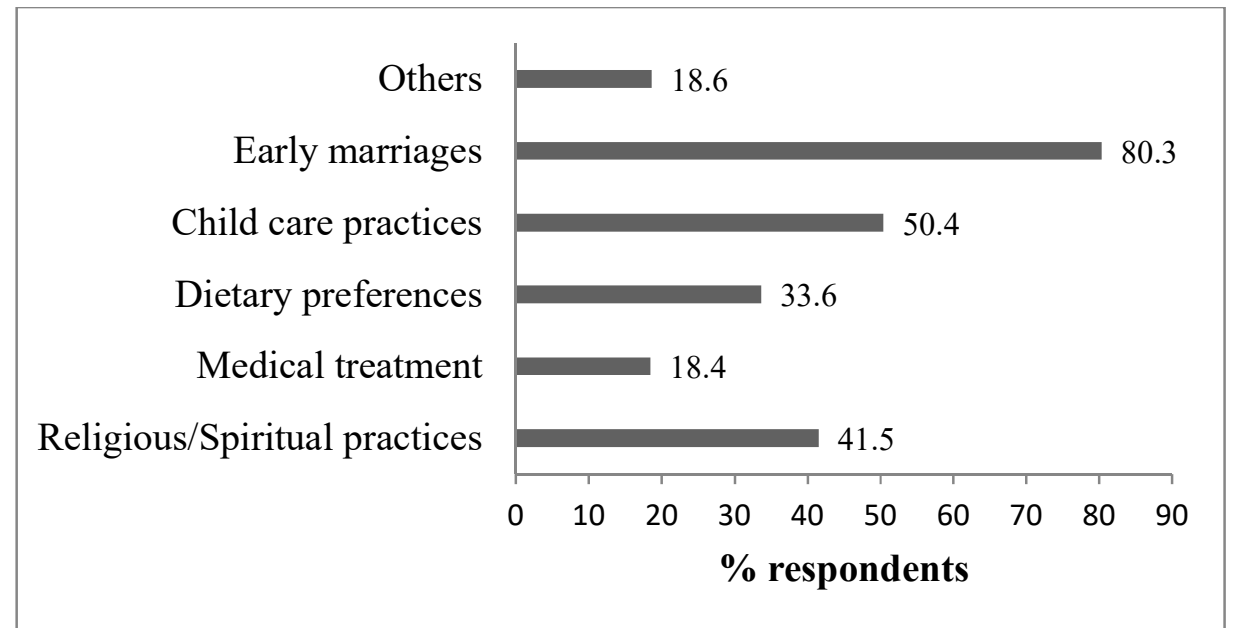

Relationship between Social cultural practices, religious/spiritual practices between and Nutrition status

The study showed significant association between Stunting and Wasting (Chi-square; $\mathrm{P}=0.048$ ); $\mathrm{X}^{2}=6.093$ ) and (Chi-square; $\mathrm{P}=0.001) ; \mathrm{X}^{2}=40.011$ ). The social cultural practices on child care practices have also been significantly associated to Underweight (Chi-square; $\mathrm{P}=0.013) ; \mathrm{X}^{2}=8.736$ ). Early marriages are significantly associated to Wasting and Stunting in their children (Chi-square; $\mathrm{P}=0.001$ ); $\mathrm{X}^{2}=48.104$ ) and (Chi-square; $\mathrm{P}=0.029$ ); $\left.\mathrm{X}^{2}=7.077\right)$ respectively. Introduction of complementary feeds in the study was significantly associated with wasting and Underweight (Chi-square; $\mathrm{P}=0.016) ; \mathrm{X} 2=8.209$ ), (Chi-square; $\mathrm{P}=0.023$ ); $\mathrm{X}^{2}=7.511$ ) respectively. The study showed that house whereby fathers play lesser role in child feeding is significantly associated with wasting (Chi-square; $\mathrm{P}=0.001) ; \mathrm{X}^{2}=40.011$ ) and where grandmother plays greater role then child complementary feeding was associated to wasting (Chi-square; $\mathrm{P}=0.001$ ); $\mathrm{X}^{2}=49.412$ ). Early introduction of Complementary feeding is significantly associated wasting and underweight (Chi-square; $\mathrm{P}=0.001$ ); $\mathrm{X}^{2}=14.326$ ). Early introduction of Complementary feeding is significantly associated wasting (Chi-square; $\mathrm{P}=0.001$ ); $\mathrm{X}^{2}=14.326$ ) and (Chi-square; $\left.\mathrm{P}=0.001) ; \mathrm{X}^{2}=20.373\right)$. Cultural barriers to Complementary feeding and distance to the market is significantly associated to wasting and underweight (Chi-square; $\mathrm{P}=0.001) ; \mathrm{X}^{2}=17.811$ ) and (Chi-square; $\left.\mathrm{P}=0.026\right) ; \mathrm{X}^{2}=7.315$ ) respectively. The study also showed that lack of information is significantly to wasting and underweight (Chi- 
square; $\left.\mathrm{P}=0.001) ; \mathrm{X}^{2}=17.041\right)$ and (Chi-square; $\left.\left.\mathrm{P}=0.001\right) ; \mathrm{X}^{2}=20.250\right)$ respectively. The presence of other children is significantly associated to wasting (Chi-square; $\mathrm{P}=0.014) ; \mathrm{X}^{2}=8.592$ ).

Table 2 Relationship between socio-cultural factors and Nutrition status

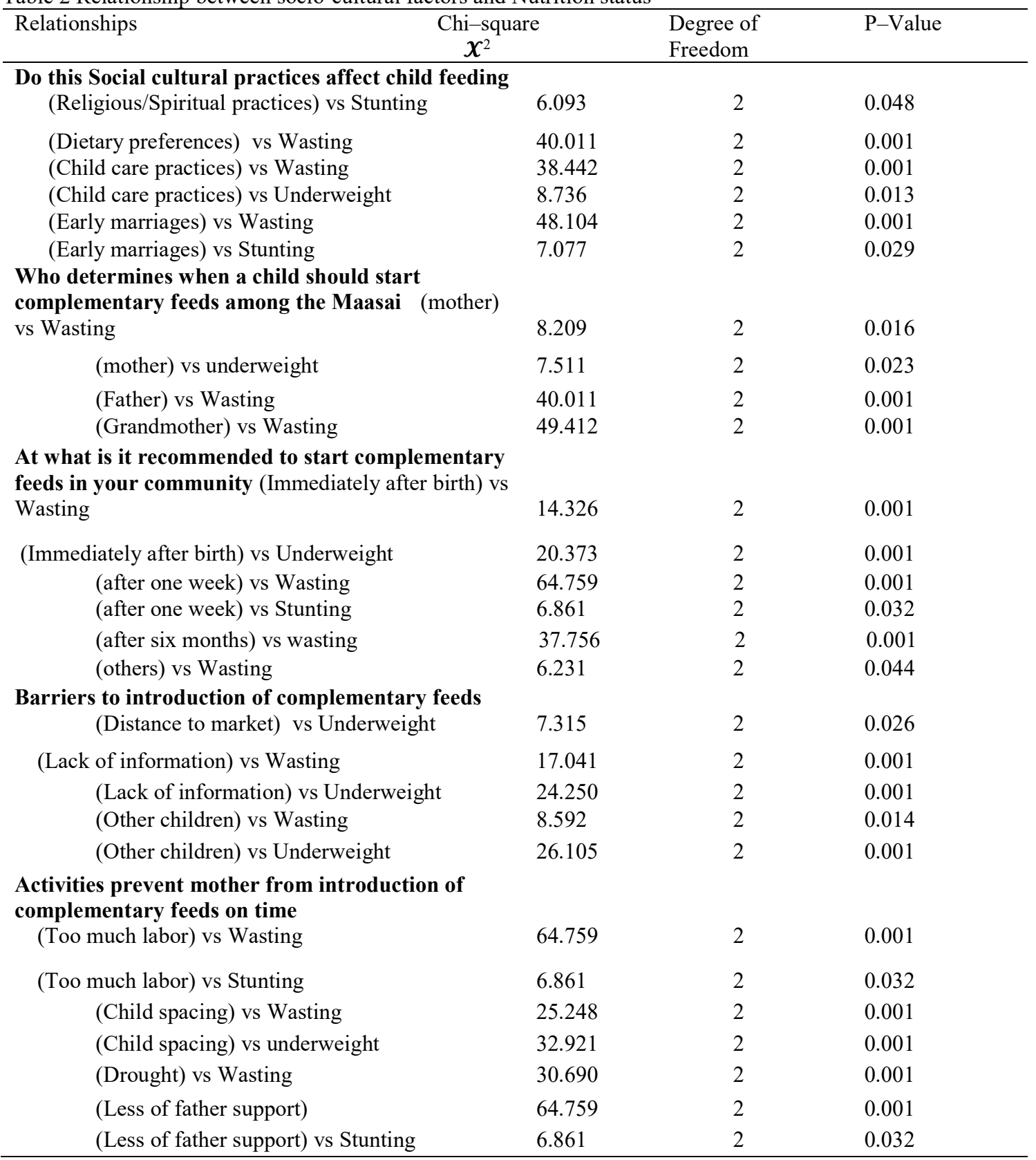

\section{Discussion}

Strong cultural factors like parent's influence have been associated to early introduction of complementary feeding and nutrition status. Some of the negative attitudes expressed by some mothers were embedded in cultural beliefs and practices. "If a woman breastfeeds while pregnant, her milk is toxic and can make the baby sick" $\left(\mathrm{M}_{2} \mathrm{FGD}_{3}\right.$ 2016).

Similar findings were noted in studies done in Brazil where older women have influence on their children in terms of exclusive breastfeeding and complementary feeding (Dias de Oliveira et al., 2014). Cultural practices seem to have dominated in this study whereby most children who were malnourished were girls, stunting was higher in 
girls than boys meaning girls have experienced long time deprivation of foods than boys the girls were also found to be underweight. (33.3\% and 14.1\%) this was not only associated to the Maasai cultural practices but attributed to prolonged draught. $\left(\mathrm{M}_{3} \mathrm{FGD}_{3} 2016\right)$, "Boys are regarded superior to girls because they don't get married but continue to sire children of continuation of family," another mother had this to say, $\left(\mathrm{M}_{2} \mathrm{FGD}_{2} 2016\right)$, "I have bore several girls for my husband but he has never slaughtered his animals for me yet when I gave him a boy I never lacked anything to eat," another said, $\left(\mathrm{M}_{5} \mathrm{FGD}_{3} 2016\right)$, "I can't blame anybody if my child is not healthy, this draught has been too much and their absolutely nothing to feed our children.

Additionally, the inadequate childcare practices (Early introduction of complementary foods, inadequate breastfeeding practices and exposure to unsanitary conditions), combined with the interaction of infection can lead to poorly nourished children. Recurrent infectious diseases reduce appetite, increase metabolic requirements, and increase nutrient loss (Caulfield et al. 2006). Stunting levels are even higher in girls compared with the boys possibly due to the emphasis placed upon boys in the community with girls not highly valued as boys. This type of belief may mean that the boy child is favored and well taken care of than the girl including feeding. This finding highlights the importance of health professionals being more cognizant of sociocultural barriers that impede healthy child-feeding practices, including inability to purchase the required food, to cater for the child nutrition status.

The prevalence of wasting is at (14.1\%) in this community. Child wasting primarily reflects severe short term deprivation of food in a child's immediate nutritional history, for example during episodes of disease such as diarrhea or in times of food shortage. In this study $62 \%$ of the children reportedly had suffered one or more form of illness in the two weeks preceding the survey.

\section{Conclusion}

The study has established the relationship between child feeding practices, socio-cultural and nutritional status of the children in the study area. The following conclusions can be drawn from the analysis of the results of the study. Caregivers in the study area seem to be knowledgeable about the recommended child feeding practices. However, the actual practice of the recommendations is seriously lacking due to perceived socio- cultural practices such as the role of the women in child caring and the general household chores as well as the limited role women in this community play in decision making regarding child feeding.

Child malnutrition is widespread in the community with more than half of the children being stunted. The high malnutrition levels are associated with poor hygiene practices, inadequate breastfeeding practices and inadequate complementary feeding practices.

The challenges to adequate complimentary feeding in the community include cultural practices, financial constraints and lack of knowledge on latest IYCF guidelines.

\section{Acknowledgements}

My gratitude's and honor is to the almighty God who has given me a chance to live and strength to do this work. I do sincerely recognize and thank the following persons who deserve credit in promoting my academic achievement.

I salute both my Supervisors Dr. Sophie Ocholah and Dr. Irene Ogada from Kenyatta University and St. Francis Xavier University who continuously supported me throughout my research process. Your kind cooperation and strong commitment has enabled me reach this far.

My appreciation and gratitude to Maasai Mara University for the study leave.

My sincere tribute to Prof Mary Walingo, and Dr. Lukaria of Maasai Mara University for their consistent help. I would like to extend my deepest gratitude to all who helped during data collection. My appreciation goes to my data analyst Dr. Kiplamai and my research assistant Mr Alex and Mr Kirui. I thank all respondents for their genuine responds during interviews.

Finally I acknowledge the Financial support of Prof. Godrick Bulitia who enabled publish this work.

\section{Funding}

None

\section{Availability of data and materials}

The data supporting the conclusions of this article is included within the article is included (and its additional file). Authors' contributions

All stated authors (SMK, SO, IAO) were involved in the study from the inception to design, acquisition of data, analysis, interpretation and drafting of the manuscript. All authors read and approved the final manuscript.

\section{Conflict of interest}


Author declares that there is no conflict of interests.

\section{Consent for publication}

Not applicable

\section{Ethics approval and consent to participate}

Permission to conduct the study was obtained from Kenyatta University Graduate School gave authority to conduct the study. Ethical and logistical clearance was sought from Kenya University Ethical Clearance Committee. The Permit to do the research was obtained from National Commission for Science, Technology and Innovation (NACOSTI). Permission was sought from County Commissioner and authority to conduct the research was also sought from County Director of Education. The researcher sought thumb printed informed voluntary consent of the respondents and participants were briefed on the research procedures and assured of confidentiality of information collected, this was done by using codes instead of names. Potential benefits of the study to the community were articulated clearly and unambiguously. No risks or harmful effects were recorded during the study since no substances were ingested or administered to the participants. Research participants were considered partners, not research subjects and the community will be informed of study results. Those found malnourished were counseled and referred to the health facility nearby.

\section{References}

1. Abdibari, M. Dereje, B. Melaku, S. Tolossa, D. Mohammed, Y. and Kiros, G, (2016). Magnitude and factors associated with malnutrition in children 6-59 months of age in Shinille Woreda, Ethiopian Somali regional state: a cross- sectional study.

2. ACC/SCN, (2016). The sixth Report on the world nutrition situation. Geneva, Switzerland.

3. Ana Cristina Lindsay, Marcia T. M., Katarina M. Sussner, Cary K. Hardwick and Karen E. Peterson (2008). Infant-feeding practices and beliefs about complementary feeding among low- income Brazilian mothers: a qualitative study.

4. Arimond, M., Daelmans, B., and Dewey, K.G. (2008). Indicators for feeding Practices in children. Lancet, 371:541-542.

5. Atchan, M., Fourer, M., \& Davis, D. (2011). The decision not to initiate breast feeding practices, women reasons, attitudes and influencing factors, a review of literature. Breastfeed review, 19 (2), 9-17.

6. Ben Slama, F., Ayari, I., Ouzini, F., Belhadj, O., \& Achour, N. (2010). Exclusive breastfeeding and mixed feeding: knowledge, attitudes and practices of primiparous mothers. Eastern Mediterranean health journal, 16 (6), 630-635.

7. Bhan, R. (2010). Complementary feeding. Indian Journal for the practicing Doctor, 1 (1), 2004-10.

8. Bhutta, Z. A., Hafeez, A., Risvi, A., Ali, N., Khan, A., Ahmad, F., Jafarey, S.N. (2013). Reproductive, maternal, newborn, and child health in Pakistan: challenges and (9884), 2207-2218. Doi:10.1016/S0140-6736 opportunities. The Lancet, 381

9. Bowman, B.A \& Russell, R.M. (2001). Present Knowledge in Nutrition. $8^{\text {th }}$ Ed, International Life Sciences Institute: Washington, Dc.

10. Central Bureau of Statistics, Kenya Demographic and Health Survey, (2003) and (2008).

11. Chelimo, F.L. (2008). Assessment of complementary feeding practices and nutritional status among children in Athi-river, Machakos District Kenya. Kenyatta University

12. County health office, (2014). Narok County Hospitals Statistical Report. Narok, Kenya: Narok County Health Statistics Office (unpublished).

13. Dewey KG, Cohen RJ, and Rollins NC. Feeding of non-breastfed children 6-24 months of age in developing countries. Food and Nutrition Bulletin 25, 2004, 377-402.

14. Dewey, K.G., \& Brown, K.H. (2003). Update on Technical Issues Concerning Complementary Feeding of Young Children in Developing Countries and Implication for Intervention Programmes. Food and Nutrition Bulletin 24 (1), 5-28.

15. FANTA \& AED (Working group on Infant and Young Child Feeding indicators). (2007). Developing and validating simple indicators of dietary quality and energy intake of infants and young children in developing countries: Additional analysis of 10 data sets. Washington, DC, USA.

16. Friedman N., Zeiger R., The role of breastfeeding in the development of allergies and asthma, (2008). Journal of Allergy and Clinical Immunology volume $115 \mathrm{pg} 1238$.

17. Gibson, R.S., Ferguson, E.L and Lehrfeld, J. (1998). Complementary Foods for Infants Feeding in Developing Countries. Dunedin, New Zealand.

18. Jens Finke, (2007). Traditional Music and Cultures of Kenya. Frankfurt University Germany.

19. Jones, G., Stekette, R.W., Black, R.E., Bhutta, Z.A., \& Morris, S.S. (2003). How many child deaths can we prevent this year, Lancer Journal of Clinic Nutrition; 362: 65-71.

20. Kabba, M.M. (2005). Breastfeeding Practices among Women. (15-49 years). A case study of Kibera Slum Nairobi; Kenya; AMREF, Kenya. 
21. Karigi, L.N. Mutuli, L. A. Bukhala P. (2016). Socio-Cultural Practices and Beleifs influencing infant and child feeding in Lubao Sub-Location Kakamega Country. Journal of nutritional Health and food engineering.

22. Kasimba, S.N. (2013). Dietary diversity and nutritional status of children (6-23) months in Makindu division, Makueni County, Kenya. Kenyatta University library.

23. Kenya National Bureau of Statistics, (KNBS \& ICF Macro (2010). Kenya Demographic and Health Survey 2008-09. Calverton, Maryland: KNBS and ICF Macro.

24. Kenya National Bureau of Statistics, (2014). Kenya Population and Housing Census; Population distribution by administrative units. Nairobi. Kenya.

25. Khanal, V., Sauer, K., Zhao, Y., (2011). Determinants of Complementary feeding practices among Nepalese children aged 6-23 months: Findings from demographic and health survey, Western Australia. Australia.

26. Kimani-Murage, E.W., Madise, N.J., Fotso, J., Kyobutungi, C., Mutua, K.M., Gitau, T.M, and Yatich N. (2011). Patterns and determinants of breastfeeding and complementary feeding practices in urban informal settlements, Nairobi Kenya. $\quad$ Biomedical Central Public Health Journal, 11:396.

27. Korir, K.K., (2013) Determinants of complementary feeding practices and nutritional status of children 6-23 months old in Korogocho slum, Nairobi County, Kenya. Kenyatta University library.

28. Makau, M.N. (2013). Feeding practices and nutritional status of children aged (0-59) months accompanying incarcerated mothers in selected women's prisons in Kenya. Kenyatta University library Kenya.

29. McDonald, C. M., Kupka, R., Manji, K. P., Bosch, R., Aboud, S., \& Kisenge, R. (2012) Predictors of stunting, wasting and underweight among Tanzanian children born to HIV-infected women. European Journal of Clinical Nutrition, 66 (11), 1265-1276.http://doi.org/10.3945/jn.111.148874

30. Ministry of Public Health and Sanitation, (2010). National Strategy on Infant and Young Child Feeding.2007-2010.Government printer.

31. Murage E., Madise N.J. Jean- Christopher F., Catherine K., Martin M.K., Gitau T.G \& Nelly Y. (2011). Patterns and determinants of breastfeeding and complementary feeding practices in urban informal settlements, Nairobi Kenya BMC Public Health Journal, 11:396 c/o: 10:118 6/1471-245811-396.

32. Mugenda, O.M., and Mugenda, A.G. (2003). Research Methods; Quantitative and Qualitative Approach. Nairobi: African Centre for Technology Studies.

33. Mugo, G. M., (2008). Influence of maternal nutrition knowledge on infant: feeding practices among the Maasai community in Narok District, Kenya. University of Nairobi.

34. Norris, F.J., Larkin, M.S., Williams, C.M., Homton, S.M., and Morgan, J.B., (2002). Factors affecting the Introduction of Complementary Foods in the Preterm Foods in the Preterm Infant. Journal of Clinic Nutrition Vol. 56, No. 5pp 448-454.

35. Ochola, S. A. (2008). Evaluation of two counseling strategies promoting exclusive breastfeeding among HIV- negative mothers in Kibera slum, Nairobi, Kenya: A randomized controlled trial. PhD Thesis. Stellenbosch University, South Africa.

36. Parada, C.M., Carvalhaes, M.A., \& Milena, T. J (2007). Complementary Feeding Practices to Children during their Year of Life. Revista Latino-Americana Enfermagem, 15 (2), 282-289.

37. Rucksana H, Asma, Iqbal K and Demissie H (1996). Early Complementary feeding is associated with low nutritional status of young infants recovering from Diarrhea Bangladesh. Journal of Clinic Nutrition

38. Theron, M., Amissah, A., Kleynhans, I. C., Albertse, E., MacIntyre, U. E. (2007). Inadequate dietary intake is not the cause of stunting amongst young children living in an informal settlement in Gauteng and rural Limpopo province in South Africa: The Nutri Gro study. Journal of Public Health Nutrition; 10 (4):379-89.

39. United Nations Administrative Committee on coordination sub-committee on Nutrition. (ACC/SCN) (2004). Fifth Report on the World Nutrition situation, Geneva.

40. UNICEF (2011). Infant and Young Child Feeding. Program guide.

41. USAID/ UNICEF/ WHO, (2010). Indicators for Assessing Infant and Young Child feeding Practices, part 3, County Profiles, WHO Press, Geneva, 11-23.

42. Wamani, H., Astrom, A.N., Peterson, S., Thorkild T., T., Tumwine, J.K. (2005). Infant and Young Child Feeding in Western Uganda: Knowledge, Practices and Social economic correlates. Journal of Tropical Pediatrics, 51 (6) 356-361.

43. WHO (2010). Indicators for Assessing Infant and Young Child Feeding Practices part 2: Measurement. WHO library cataloguing-in-publication data. Dept of Child and Adolescent Health and Development. ISBN 9789241599290 (NLM classification: WS 120). 
44. WHO (2002). Complementary feeding: report of the global consultation and summary of guiding principles for complementary feeding of the breastfed child. Geneva Switzerland.

45. WHO/UNICEF/USAID (2004). National guidelines on infant and young child feeding practices. WHO press. Geneva.

46. Wood, C.H., Glanville, H. de and Vaughan, J.P. (2008). Community Health. AMREF Nairobi, Kenya.

47. www. Kenya-information guide.com 2014, October, 1400. 